\title{
AO Assisted NIR 3D Spectroscopic Imaging - Can an ELT See the Earth at 10pc?
}

\author{
Markus Feldt ${ }^{1}$, Alessandro Berton ${ }^{1}$, Thomas Henning ${ }^{1}$, \\ Raffaele Gratton ${ }^{2}$, Massimo Turatto ${ }^{2}$, H. M. Schmid ${ }^{3}$, M. Kasper ${ }^{4}$ \\ and J. L. Beuzit ${ }^{5}$ \\ ${ }^{1}$ Max Planck Institute for Astronomy, Königstuhl 17, D-69117 Heidelberg, Germany \\ email: mfeldt@mpia.de \\ ${ }^{2}$ Osservatorio Astronomico di Padova, Vicolo dell'Osservatorio 5, I-35122 Padova, Italy, \\ email: gratton@astro.pd.it \\ ${ }^{3}$ Institut für Astronomie, ETH Zentrum, CH-8092 Zürich, Switzerland \\ email: schmid@astro.phys.ethz.ch \\ ${ }^{4}$ European Southern Observatory, Karl-Schwarzschild-Straße 2, D-85748 Garching, Germany \\ email: mkasper@eso.org \\ ${ }^{5}$ Laboratoire d'Astrophysique, Observatoire de Grenoble, BP 53, \\ F-38041 Grenoble Cédex 9, France email: Jean-Luc.Beuzit@obs.ujf-grenoble.fr
}

\begin{abstract}
The European southern observatory ESO is currently undertaking the ambitious task of building a "Planet Finder" instrument for the VLT. The concept for this instrument includes a 3D spectroscopic imager assisted by a very powerful eXtreme $\mathrm{AO}$ system. In one of the two phase-A studies for this project, we have developed a simulation software for this kind of instrument which has now been extended and applied to the case of various ELTs. In this presentation, we give results of simulations and discuss achievable signal to noise ratios and prospects for detection and characterization from young gas giants down to terrestrial type planets. ELTs of various diameters are considered as well as a set of environmental conditions.
\end{abstract}

Keywords. instrumentation: spectrographs, methods: data analysis, techniques: high angular resolution, techniques: spectroscopic, (stars:) planetary systems.

\section{Introduction}

It is not long ago that astronomers believed it would always remain impossible to actually get an image of a planet that is not part of our own solar system. Nowadays, a number of project is dedicated to devise instruments that will achieve exactly that. ESO is planning to put a "Planet Finder" instrument on the VLT (Beuzit et al. 2005), and the GEMINI observatory has a similar project (Macintosh et al. 2004). In the context of the CHEOPS project, which was one of the two phase-A studies conducted in the context of the ESO Planet Finder, we devised the concept of using a 3D integral field spectrograph (IFS) as a sophisticated differential imaging device in order to suppress the residual speckle halo from a bright star and eventually to directly detect extrasolar planets (Feldt et al. 2004). As a result of this study, we found that such a device, supported by an eXtreme AO (XAO) system will, when placed on the VLT, be capable of detecting rather young $(10 \mathrm{Myr})$ Jupiter-sized $\left(M=5 \mathrm{M}_{\mathrm{Jup}}\right)$ planets in orbit around stars up to $40 \mathrm{pc}$ distant (Berton et al. 2005b). Older Planets would have to be slightly larger than Jupiter to be detected at close distances $(\sim 5 \mathrm{pc})$

Not satisfied with such prospects, astronomers have already planned the next generation of such instruments for a new generation of "extremely large telescopes" (ELTs), the 
Table 1. Values used in the simulation of the atmosphere.

\begin{tabular}{rl}
\hline No. of Layers & 1 \\
Fried radius $r_{0}$ & $0.17 \mathrm{~m}$ \\
Coherence time $\tau_{0}$ & $3 \mathrm{~ms}$ \\
Wind speed $v_{\text {wind }}$ & $17.7 \mathrm{~m} / \mathrm{s}$ \\
Outer scale $L_{0}$ & $25 \mathrm{~m}$ \\
\hline
\end{tabular}

most prominent example of which is ESO's "OverWhelmingly Large telescope (OWL)" with a primary mirror diameter of $100 \mathrm{~m}$. The science case in the area of finding planets is for these telescopes and their instruments no longer limited to young giant planets, but explicitely includes the detection of Jupiter twins, and, more challenging and interesting, terrestrial planets (Verinaud et al. 2005). All science cases include not only direct imaging detections, but also characterization of the detected planets, e.g. by spectroscopy and/or polarimetry.

In order to evaluate the feasibility of such science cases and to estimate the observation times required to achieve these challenging goals, we applied our simulation code to the case of two ELTs hypothetically equipped with an extreme AO system and an integral field spectrograph basically identical to the one devised in the context of CHEOPS (Claudi et al. 2004).

\section{Instrument and Simulation Code}

A basic description of the IFS can be found in Claudi et al. 2004. In the context of CHEOPS, and still for "Planet Finder", the IFS consists of an array of $254 \times 254$ micro lenses in the focal plane covering a field-of-view of $3 . .5 \times 3 . .5$, followed by an Amici prism and a re-imaging optics that forms spectrally resolved $(R \sim 15)$ micro-pupil images on the $2 k \times 2 k$ detector. The system is placed behind a powerful XAO system with $\sim 1600$ actuators, running at loop frequencies between $1 \mathrm{kHz}$ and $2 \mathrm{kHz}$. A description of the full instrument is given in Beuzit et al. (2005).

The simulation code is described briefly in Berton et al. (2005a) and in more detail in Berton et al. (2005b).

\section{Simulation Set-up}

The simulation approach chosen is a modular structure that propagates phase and amplitude arrays from one module to the next one, modifying it according to the physics involved. The discrete time step of the simulation is $1 \mathrm{~ms}$, phase screens are generated and propagated in in a successive way in order to allow for the correlated nature of the speckle noise.

\subsection{Atmosphere}

The atmosphere is simulated by creating a continuous series of phase screens. the basic parameters used in this simulation are summarized in Tab. 1. For the generation of each phase screen, the predecessor is shifted a bit according to the wind speed and slightly modified using a Markov process to partially de-correlate the screen from the previous one. The amplitude is assumed to be flat, since it has been shown by Masciadri, Feldt and Hippler (2004) that scintillation can be neglected for intermediate Strehl ratios. 

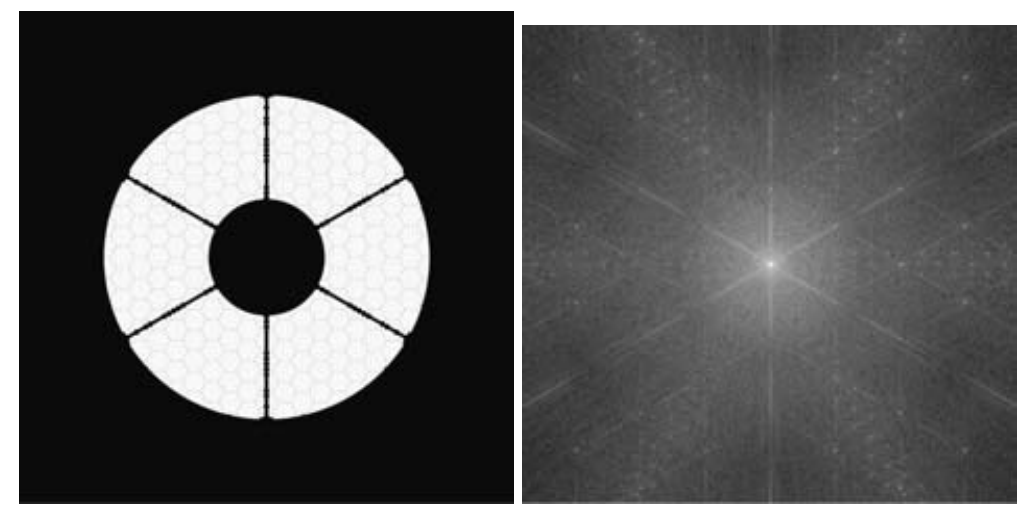

Figure 1. Shape of the OWL pupil and resulting PSF.

\subsection{Telescope}

The telescope is represented simply by its aperture which is multiplied onto the uniform input amplitude distribution. Two patterns were generated using the OWL Pupil Simulator by N. Yaitskova (priv. comm.). Two cases were simulated: A complete OWL design with a $100 \mathrm{~m}$ primary consisting of $1.6 \mathrm{~m}$ hexagonal segments, and a $26 \mathrm{~m}$ segmented secondary. Additionally, we simulated a scaled-down version with a primary diameter of $50 \mathrm{~m}$. In this case the word "scaled-down" is to be taken literally, which will probably not match a real configuration since from an engineering point of view one would desire to keep the segment diameter at $1.6 \mathrm{~m}$ rather than using every component in half size.

There are no phase errors included that originate in the telescope whatsoever, neither in nor between the segments, no vibrations, and no missing segments. The amplitude map used for the simulations can be seen in Fig. 1 (For testing the reproduction quality, try to disentangle the small primary segments from the larger secondary segments) together with the resulting PSF (in the miraculous case where no atmosphere is present above OWL).

\subsection{Adaptive Optics}

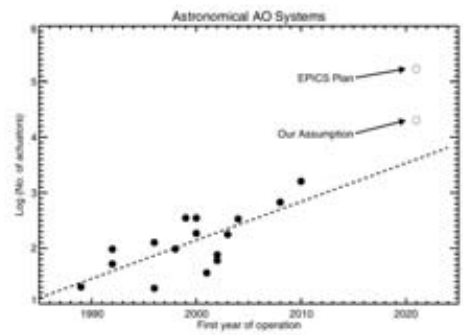

Figure 2. Growth of the number of actuators in astronomical AO systems over the years.

The AO system is simulated in a very simple way.: The atmospheric phase screens are down-sampled (using averaging) to the assumed sensor element resolution. This reexpanded version of the down-sampled phase screen is then subtracted from the atmospheric one arriving one AO loop time step (2 ms) later to provide the corrected phase map. This assumes an identical sampling for the wavefront sensor and the deformable mirror and automatically rules out all sources of noise (measurement noise in the WFS, aliasing, etc.). The two only errors included in this way are the temporal error and the 
fitting error. The two single parameters describing the system are the number of actuators $(20,000)$ and the correction frequency $(500 \mathrm{~Hz})$.

It has been argued during the conference that the choice of the number of actuators is rather unsuitable, particularly since the resulting control radius is $r_{c}=\frac{\lambda}{2 d} \approx 0$ '. 14 and thus most of the planets we are trying to detect are outside this radius in the (speckle)noisy seeing halo that is not controlled by the XAO. We note however that our assumption is still quite optimistic (see also Fig. 2) and in a real system of our dimensions one would probably not notice the effect of a control radius-limited basin region in the PSF anyway.

\subsection{Integral Field Spectrograph}

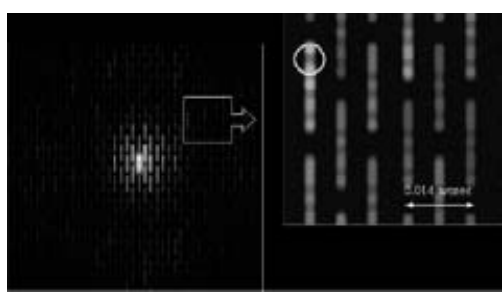

Figure 3. Spectra on the IFS detector.

The integral field spectrograph is the core module of our simulation code. This is precisely the reason why we do not discuss its details here. You can find an extended description in Berton et al. 2005a and a thorough explanation in Berton et al. 2005b. The output of the IFS module the detector image that shows the aligned spectra as seen in Fig. 3.

\subsection{Neglects}

Our simulations have been critisized for not starting systematically at the optimistic or pessimistic end of chain of effects that determines the final signal-to-noise ratio of a planetary detection.

We note that the primary goal of our simulations was to learn how the detectability of earth-like planets depends on the diameter of future giant telescopes under two keyassumptions. These key-assumptions are

(a) The AO system with which a system like the assumed one would be equipped is excellent and state of the art in about 2020, but it will very likely be far from perfect.

(b) The device for differential imaging will be a TIGRE-type IFS as suggested for the VLT Planet Finder.

Our results are to be seen in this context. Even though, simulations presented here do neglect a number of effects, the most important for which are:

- Flat fielding is not explicitly simulated, we simply multiply our pixel counts with a random number such that a residual error level of $10^{-4}$ is reproduced.

- The alignment of the spectra on the pixel grid is perfect. This will not be the case in a real system, but it will have to be achieved by sophisticated interpolation - which of course will cause additional errors. Investigation of this effect is ongoing.

- Interference between lenslets is not taken into account here as the micro pupil is calculated individually for each lenslet. We have however performed dedicated simulations with arrays of up to $40 \times 40$ lenslets that show the SNR reduction is only by a factor of $\sim 0.98$.

- Exploitation of more than one absorption feature is not simulated here, we present the simple subtraction of two wavelength. In Berton et al. 2005b we show a dedicated 
simulation which makes use of two planetary absorption features in the $J$-band using up to 9 spectral elements provided by the IFS. This improves the detection SNR by a factor of 2 .

In summary, the most important thing we have neglected in the present work is the strength of the IFS, so our results are to be seen on the pessimistic side with room for improvement by up to a factor of 2 in the detection SNR or a factor of 4 in the needed integration times presented in the following section.

\section{Results}

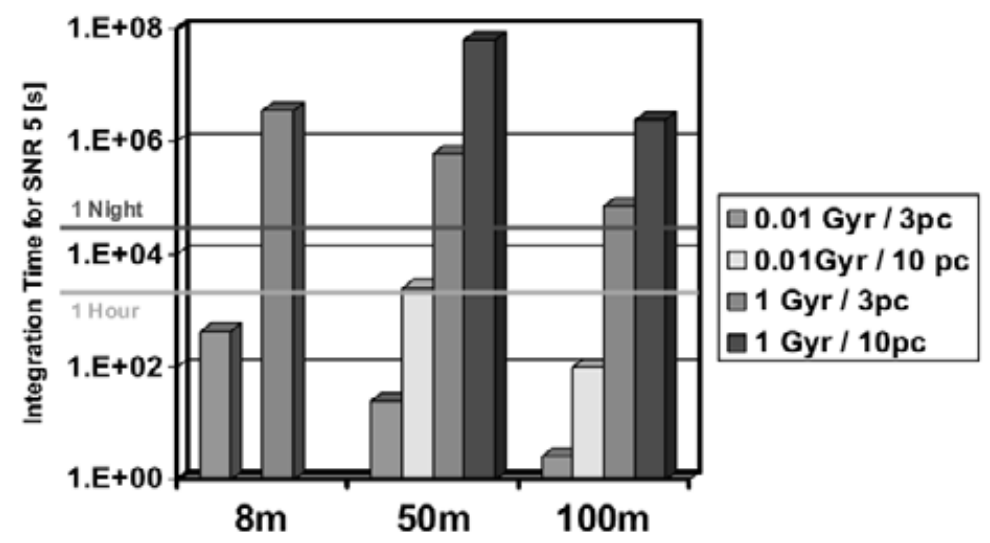

Figure 4. Integration times needed to detect a planet of 1 Jupiter mass in a 1 AU orbit around a G2V star with a SNR of 5 .

Fig. 4 gives the result of our simulations for Jupiter like planets, i.e. Planets of 1 Jupiter mass orbiting a G2 V star at 1 AU orbital radius. The times indicated are needed to achieve a detection SNR of 5 . Note that these are standard cases for comparison, not actually existing planets. Note also that the numbers quoted for " $8 \mathrm{~m}$ " were computed during the CHEOPS study. In CHEOPS, unlike in the present Planet Finder concept, it was not foreseen to observe closer than $00^{\prime \prime} 3$ from the star, which is why some planet classes are not shown for the $8 \mathrm{~m}$ case.

The single result we obtained so far for an exo-earth at $3 \mathrm{pc}$ is as follows: We applied a spectral resolution of 150 (instead of 15 for the exo-Jupiters) in order to escape saturated absorption by both planet's atmospheres and rescaled the spectra accordingly in order to compensate the transmission by earth's atmosphere. In this way we could apply differential imaging to the exactly identical features that have just been compensated. Using this technique we calculate an observing time of $1000 \mathrm{hrs}$ to detect the exo-earth in a $1 \mathrm{AU}$ orbit around a G2 V star at $3 \mathrm{pc}$.

\section{Discussion}

It is clear that giant telescopes gain an enormous advantage over $8 \mathrm{~m}$ telescopes when trying to image planets. In some cases, integration times can become so short that completely new science fields can be opened - time series photometry or even spectroscopy in fact even radial velocity measurements on exo-Jupiters in order to detect their satellites appear feasible in some cases. The detection of terrestrial planets however appears very difficult with an instrument suite as the one simulated here. However, by using the 
full advantage of an IFS - which gains a factor of 2 in SNR - and by possibly decreasing the spectral resolution by a factor of 10 and simply making use of more photons, the integration time needed might be reduced to levels that just appear achievable (of the order of 1 or a few nights - to find an exo-earth at 3 pc!!). We also note that the current EPICS study (Verinaud et al. 2005) suggests a much more sophisticated AO system plus a coronographic device which can potentially reduce the photon noise in the PSF wings. Such a device would - if the technology can indeed be developed - facilitate the detection of terrestrial planets out to distances of approximately $25 \mathrm{pc}$.

\section{References}

Berton, A., Feldt, M., Gratton, G., Henning, Th., Desidera, S., et al. 2005a, This proceedings

Berton, A., Gratton, R.G., Feldt, M., Henning, Th., Desidera, S., et al. 2005b, submitted to Astronomy and Astrophysics

Beuzit, J.-L., Mouillet, D., Moutou, C., Dohlen, K., Puget, P., et al. 2005, This Volume

Claudi, R. U., Turatto, M., Gratton, R., Antichi, J., Buson, S., et al. 2004, Proceedings of the SPIE, Vol. 5492, P. 1351

Feldt, M., Henning, Th., Hippler, S., Turatto, M., Neuhäuser, R. et al. 2004, The CHEOPS Project: CHaracterizing Exo-planets by Opto-infrared Polarimetry and Spectroscopy, in: "Exploring the Cosmic Frontier" eds. A. P. Lobanov et al., ESO Astrophysics Symposia, 2005 , in press

Macintosh, B. A., Bauman, B., Wilhelmsen Evans, J., Graham, J. R., Lockwood, C., et al. 2004, Proceedings of the SPIE vol. 5490, p. 359

Masciadri, E., Feldt, M., \& Hippler, S. 2004, Astrophysical Journal 613, P. 572

Christophe Verinaud, Hubin Norbert, Kasper Markus, Antichi Jacopo, Beuzit Jean-Luc, et al. 2005, This proceedings

\section{Discussion}

Close: Are you really claiming that at Strehl ratios of only $60 \%$ you see a cleared-out basin in the PSF centre?

We do, but this is only due to our rather perfect wavefront sensing and correction device - which is simply re-sampling the wavefront itself to the sensor and DM resolution. In this way, we of course have no aliasing whatsoever.

FRIEDLund: Note that your 1 Myr Jupiter at 3 pc does of course not exist. Not many young stars are in the vicinity of the sun.

This is true, our calculations are so far limited to a few (fictitious) "standard" cases that were agreed upon in order to compare results of the two "Planet Finder" studies conducted by ESO. 